\title{
Primary gastric squamous cell carcinoma in situ originating from gastric squamous metaplasia
}

Primary squamous cell carcinoma (SCC) is extremely rare in the stomach [1]. Although there is only one published case of detection at an early stage [2], all reported

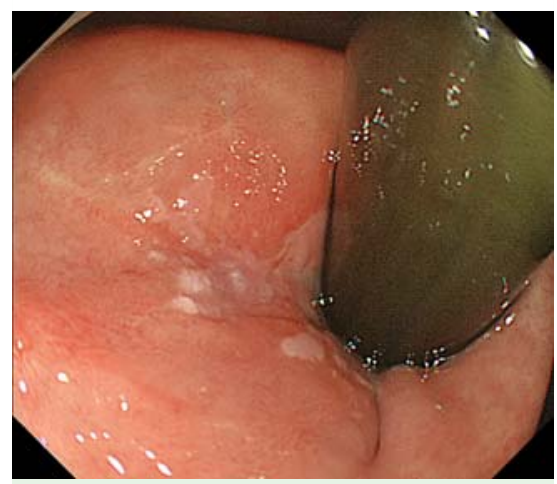

Fig. 1 A conventional endoscopy revealed an irregular whitish superficial depressed lesion in the lesser curvature of the cardia.

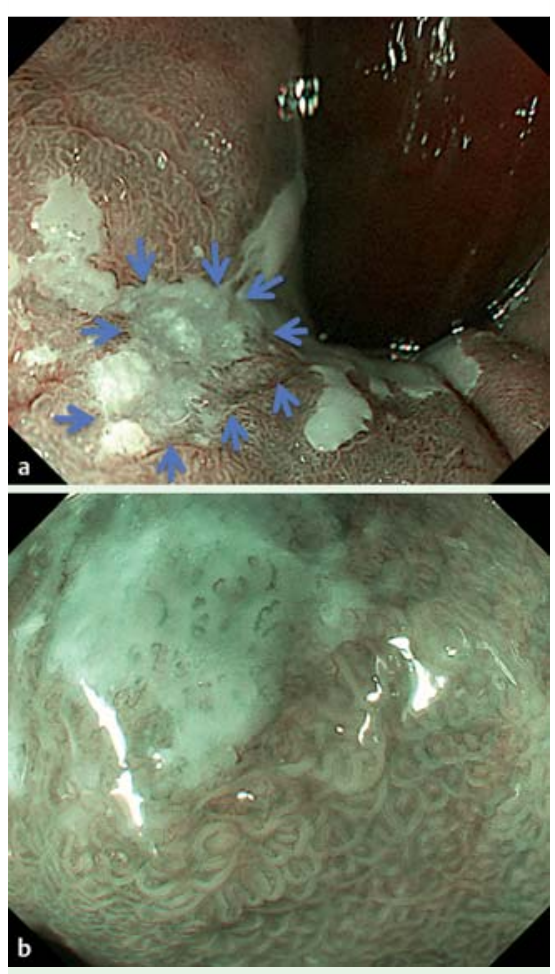

Fig. 2 Narrow-band imaging (NBI) of the lesion. a A well demarcated white-brownish area, $8 \mathrm{~mm}$ in size, which was different from the surrounding normal gastric mucosa was seen without magnification b Magnifying NBI endoscopy found dilated and tortuous capillaries mimicking intrapapillary capillary loop in a part of the demarcated depressive region. cases underwent surgical resection. We describe a case of SCC in situ arising from squamous metaplasia in the stomach, which was successfully treated with endoscopic submucosal dissection (ESD).

A 71-year-old man underwent surveillance endoscopy after endoscopic treatment for early esophageal cancer. A conventional endoscopy revealed a whitish lesion of irregular shape in the cardiac region ( Fig. 1).

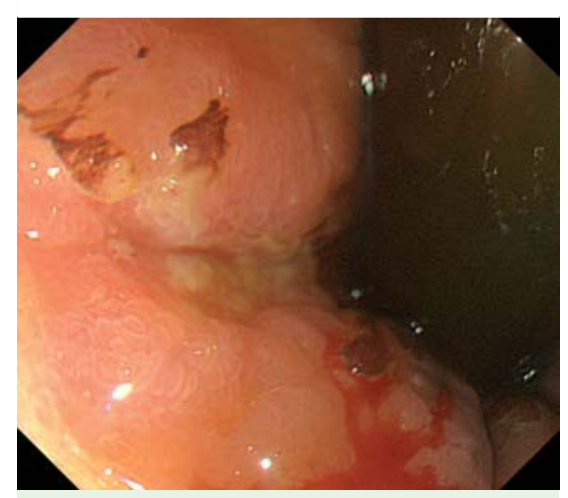

Fig. 3 With chromoendoscopy using 3.0\% Lugol solution, the white-brownish area was shown as a Lugol-unstained area, partly surrounded by a small Lugol-stained area.
The lesion was partly contiguous to the esophageal mucosa at the esophagogastric junction. The endoscopic findings of narrow band imaging (NBI) without magnification showed a well demarcated white-brownish area, $8 \mathrm{~mm}$ in size, which was different from the surrounding normal gastric mucosa ( $\bullet$ Fig. 2 a).

Dilated and tortuous capillaries in a part of the demarcated depressive region were detected by magnifying NBI endoscopy ( Fig.2b). On chromoendoscopy using 3.0\% Lugol solution, the whitebrownish area remained unstained, with a small portion of Lugol staining around this area ( $\bullet$ Fig. 3 ).

The histological findings of the biopsy specimen suggested SCC. The lesion was removed by ESD ( $\bullet$ Video 1 ).

The resected specimen showed a whitish superficial depressed area, about $8 \mathrm{~mm}$ in size ( $\bullet$ Fig. 4).

\section{Video 1}

The lesion was removed by endoscopic submucosal dissection.

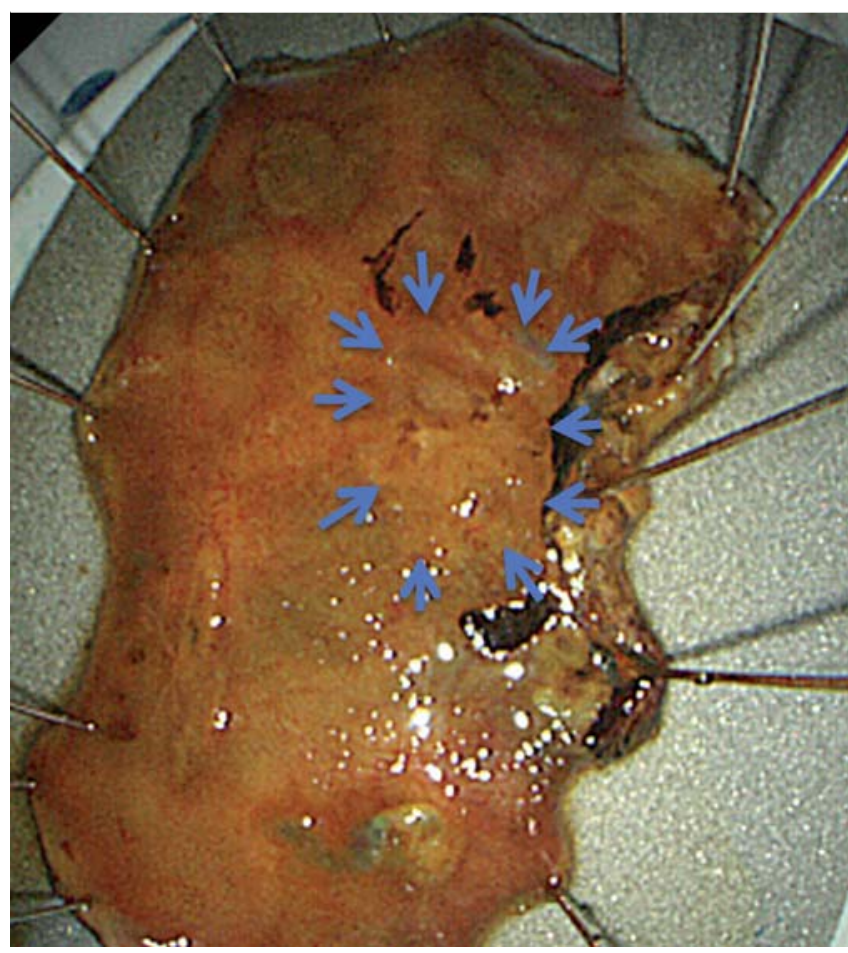

Fig. 4 The resected specimen identified a whitish superficial depressed area, about $8 \mathrm{~mm}$ in size, which was not stained with Lugol solution. The Lugolunstained depressed region was partly surrounded by Lugolstained mucosa. 


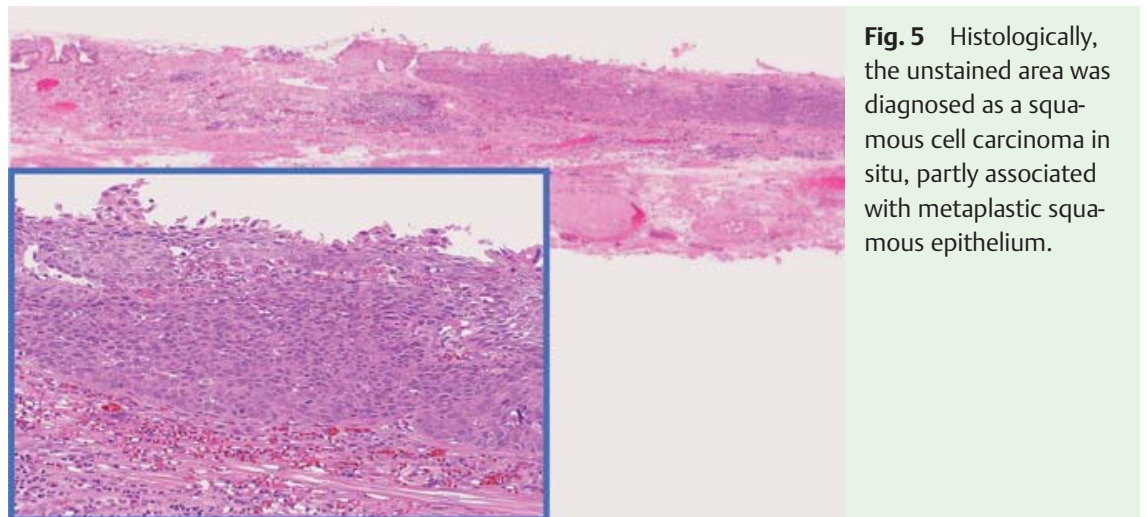

Histologically, the unstained area was diagnosed as an SCC in situ associated with non-cancerous squamous epithelium ( $\bullet$ Fig. 5), with no cancer cells in the resected margin.

In the present case, the tumor was associated with non-cancerous squamous epithelium partly connecting to the esophageal mucosa, and most of the lesion was surrounded by gastric mucosa. Moreover, at the periphery of the tumor lesion, small insular areas of the mucosa were lined with metaplastic squamous epithelium, without glandular structure or esophageal glands. Thus we postulated that the SCC originated from the squamous metaplastic epithelium derived from the gastric columnar epithelium. Squamous metaplasia in the proximal stomach is often associated with an injurious process such as gastroesophageal reflux or gastric inflammation [3-5]. Although rarely encountered, we should bear in mind that cancers can occur in this kind of squamous metaplasia, as early detection offers more opportunity for less-invasive endoscopic treatment.
Competing interests: None

Endoscopy_UCTN_Code_CCL_1AB_2AD_3AB

Y. Oono ${ }^{1}$, K. Fu르, E. Nagahisa ${ }^{1}$,

T. Kuwata ${ }^{3}$, H. Ikematsu ${ }^{1}$, T. Yano ${ }^{1}$,

T. Kojima ${ }^{1}$, K. Minashi ${ }^{1}$, S. Fujii ${ }^{4}$,

A. Ochiai ${ }^{4}$, K. Kaneko ${ }^{1}$

1 Division of Digestive Endoscopy and Gastrointestinal Oncology, National Cancer Center Hospital East, Chiba, Japan

2 Department of Gastroenterology, Juntendou University Nerima Hospital, Tokyo, Japan

3 Pathology Section, Clinical Laboratory Division, National Cancer Center Hospital East, Chiba, Japan

4 Pathology Division, Research Center for Innovative Oncology, National Cancer Center Research Institute East, Chiba, Japan

\section{References}

1 Straus R, Heschel S, Fortmann DJ. Primary adenosquamous carcinoma of the stomach. A case report and review. Cancer 1969; 24: 985-995

2 Takita J, Kato H, Miyazaki T et al. Primary squamous cell carcinoma of the stomach: a case report with immunohistochemical and molecular biologic studies. Hepatogastroenterology 2005; 52: 969-974

3 Fass R, Sampliner RE. Extension of squamous epithelium into the proximal stomach: a newly recognized mucosal abnormality. Endoscopy 2000; 32: 27-32

4 Takeda H, Nagashima R, Goto $T$ et al. Endoscopic observation of squamous metaplasia of the stomach: a report of two cases. Endoscopy 2000; 32: 651-653

5 Fass R, Garewal HS, Hayden CW et al. Preferential repair by squamous epithelium of thermal induced injury to the proximal stomach in patients undergoing ablation of Barrett's esophagus. Gastrointest Endosc 2001; 53: $711-716$

\section{Bibliography}

DOI $10.1055 / \mathrm{s}-0030-1255807$

Endoscopy 2010; 42: E290 -E291

(c) Georg Thieme Verlag KG Stuttgart · New York . ISSN 0013-726X

Corresponding author

\section{Y. Oono, MD}

Department of Gastroenterology

National Cancer Center Hospital East

6-5-1, Kashiwanoha

Kashiwa City

Chiba, 277-8577

Japan

Fax: +81-4-71346928

doc2adova@yahoo.co.jp 